EPJ Web of Conferences 66, 02069 (2014)

DOI: $10.1051 /$ epjconf/ 20146602069

(C) Owned by the authors, published by EDP Sciences, 2014

\title{
Gamow-Teller strength in deformed nuclei within self-consistent pnQRPA with the Gogny force
}

\author{
M. Martini ${ }^{1,2, a}$, S. Péru ${ }^{2}$, and S. Goriely ${ }^{1}$ \\ ${ }^{1}$ Institut d'Astronomie et d'Astrophysique, CP-226, Université Libre de Bruxelles, 1050 Brussels, Belgium \\ ${ }^{2}$ CEA,DAM, DIF, F-91297 Arpajon, France
}

\begin{abstract}
In recent years fully consistent quasiparticle random-phase approximation (QRPA) calculations using finite range Gogny force have been performed to study electromagnetic excitations of several axially-symmetric deformed nuclei up to the ${ }^{238} \mathrm{U}$. Here we present the extension of this approach to the charge-exchange nuclear excitations (pnQRPA). In particular we focus on the Gamow-Teller (GT) excitations. A comparison of the predicted GT strength distribution with existing experimental data is presented. The role of nuclear deformation is shown. Special attention is paid to $\beta$-decay half-lives calculations for which experimental data exist.
\end{abstract}

Spin-isospin nuclear excitations, in particular the Gamow-Teller (GT) resonances, play nowadays a crucial role in several fields of physics. First, in nuclear physics since they can provide informations on the nuclear interaction, on the equation of state of asymmetric nuclear matter and on the nuclear skin thickness. Second, in astrophysics, since they influence stellar evolution and nucleosynthesis by governing the electroweak processes. Finally, in particle physics in connection with the neutrino physics beyond the standard model and the evaluation of the $V_{u d}$ element of the Cabibbo-KobayashiMaskawa quark-mixing matrix.

Experimentally the spin-isospin nuclear excitations are studied via charge-exchange reactions, such as $(\mathrm{p}, \mathrm{n}),(\mathrm{n}, \mathrm{p}),\left(\mathrm{d},{ }^{2} \mathrm{He}\right),\left({ }^{3} \mathrm{He}, \mathrm{t}\right)$ or $\left(\mathrm{t},{ }^{3} \mathrm{He}\right)$ and $\beta$-decay measurements. In spite of the great efforts and interest, the whole nuclear chart cannot be experimentally studied. To study the nuclei experimentally inacessible one can rely on theoretical models. In this context one of the most employed models is the so called proton-neutron quasiparticle random-phase approximation (pnQRPA). To treat consistently isotopic chains from drip line to drip line two main features of the theoretical model are in order: the possibility to deal with deformed nuclei and the use of an unique effective nuclear force. The term unique has here two meanings. First of all, it means that the interaction is the same for all the nuclei; second, that the nuclear interaction used to describe the ground state and the excited states is the same (this is the so-called self-consistency of the calculation). In spite of the relatively large number of pnQRPA calculations, only a very few of those include both features. Furthermore, even in such self-consistent calculations, it remains at least one coupling constant, typically in the particle-particle channel, which should be considered as a free parameter usually fitted to half-lives or to the experimental position of the GT excitation energy.

Here we present the fully consistent axially-symmetric-deformed pnQRPA calculation based on the finite range Gogny force. The originality of the present work consists in the use of the Gogny

\footnotetext{
a e-mail: martini.marco@gmail.com
} 


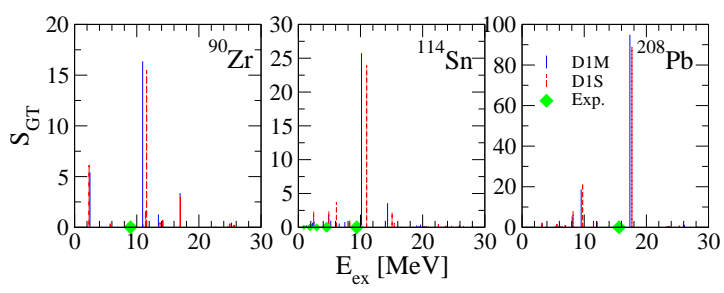

Figure 1. pnQRPA GT strength distributions in ${ }^{90} \mathrm{Zr},{ }^{114} \mathrm{Sn}$ and in ${ }^{208} \mathrm{~Pb}$ calculated the with D1M and D1S forces. The experimental data of the energy peaks position [7-9] are shown as diamonds on the $x$-axes.

force, since up to now the other self-consistent (spherical or axially deformed) calculations were performed either with a zero-range Skyrme-type force or within the relativistic covariant description. In the present approach, no additional parameters are introduced in the pnQRPA calculation beyond those characterizing the effective nuclear force (namely D1M [1] or D1S [2]). This work represents a transposition to the charge-exchange field of the fully consistent axially-symmetric-deformed QRPA calculations presented in [3] and devoted to the study of the electromagnetic excitation of deformed nuclei $[4,5]$. In this approach pairing correlations which play an important role in open shell nuclei are automatically included. The possibility to take into account the nuclear deformation is also fundamental. The $\beta$-decay properties of nuclei (including their impact on the r-process nucleosynthesis [6]) as well as the nuclear matrix elements for the double $\beta$ decay have been shown to depend significantly on the deformation parameter. Furthermore, deformed nuclei present strong fragmentation in their response functions and different nuclear shapes can be experimentally distinguished.

Our approach is based on the pnQRPA on top of axially-symmetric-deformed Hartree-FockBogoliubov (HFB) calculations. The HFB equations are solved in a finite harmonic oscillator (HO) basis. As a consequence, the positive energy continuum is discretized. The number of HO major shells included in the model space depends on the atomic mass number. All HFB quasiparticle states are included to generate the 2-quasiparticle (2-qp) excitations. This means that our calculation can be performed without cut in energy or in occupation probabilities. According to the symmetries imposed in the present axially-symmetric-deformed HFB calculation in even-even nuclei, the projection $K$ of the angular momentum on the symmetry axis and the parity $\pi$ are good quantum numbers. Consequently, pnQRPA calculations can be performed separately for each $K^{\pi}$ block. To solve the pnQRPA matrix equation we use the same numerical procedure as recently applied to the study of giant resonances of the heavy deformed ${ }^{238} \mathrm{U}$ [4]. This procedure is based on a massive parallel master-slave algorithm. The solution of the pnQRPA matrix equation provides the energies $\omega_{n}$ of the excited states of the parent nucleus and the set of amplitudes describing the wave function of the excited state in terms of the two quasiparticle excitations.

Once the pnQRPA matrix equation is solved, we can calculate the response to the Gamov-Teller operator

$$
\hat{O}_{G T}=\sum_{i=1}^{A} \vec{\sigma}(i) \tau_{-}(i)
$$

generating a spin-flip ( $\Delta S=\Delta J=1)$ response. In an axially-symmetric-deformed nuclear system, the response function of a given $J^{\pi}$ contains different $K^{\pi}=0^{\pi}, \pm 1^{\pi}, \ldots, \pm J^{\pi}$ components. In this case the GT $J^{\pi}=1^{+}$distributions are obtained by adding twice the $K^{\pi}=1^{+}$result to the $K^{\pi}=0^{+}$result. Details to go from the intrinsic to the laboratory frame can be found in [3]. 


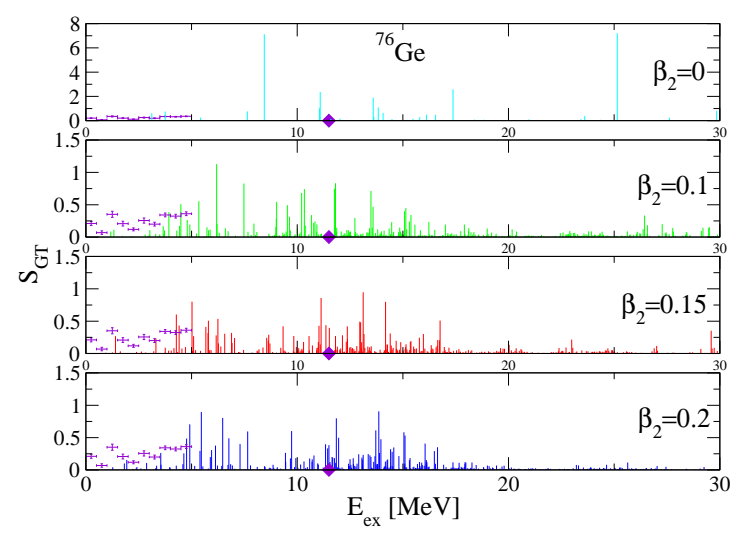

Figure 2. pnQRPA GT strength distributions in ${ }^{76} \mathrm{Ge}$ obtained with the D1M force for several values of the deformation parameter $\beta_{2}$, including the HFB ground state minimum of $\beta_{2}=0.15$. The experimental low energy data [10] as well as the energy position of the main GT peak are also shown.

We consider the closed neutron shell ${ }^{90} \mathrm{Zr}$ and the ${ }^{208} \mathrm{~Pb}$, as well as neutron open shell nucleus ${ }^{114} \mathrm{Sn}$ as test cases. Their GT strength distributions calculated with D1M and D1S interactions are shown in Fig 1. In the same figure, are shown the corresponding experimental values [7-9] for the major excitation energies obtained from $(p, n)$ scattering data. The results are expressed as a function of the excitation energy $E_{e x}$ referred to the ground state of the daughter nucleus. In our model it is obtained by subtracting the lowest two-quasiparticle energy $E_{0}$ from the excitation energy $\omega_{n}$ of the parent nucleus calculated in the pnQRPA, i.e. $E_{e x}=\omega_{n}-E_{0}$. The two interactions give quite similar results for the position of the main peak. The agreement between our calculations and experimental data is rather satisfactory. For the two interactions we have also verified that the Fermi and the Ikeda sum rules are exhausted by our strength distributions.

The above results refer to three spherical nuclei. As already emphasized, our approach described axially symmetric deformed nuclei. As an example of deformed nucleus, we show in in Fig. 2 the ${ }^{76} \mathrm{Ge}$ spin-isospin excitations, more precisely the GT distributions obtained with D1M Gogny interaction for several values of the deformation parameter $\beta_{2}$, including the HFB ground state minimum of $\beta_{2}=0.15$. Experimental data [10] are also included. As expected, the deformation tends to increase the fragmentation of the response. Calculations with different deformations produce peaks that are displaced. Deformation effects also influence the low-energy strength and consequently can be expected to affect $\beta^{-}$-decay half-lives.

In the allowed GT decay approximation, the $\beta^{-}$-decay half-life $T_{1 / 2}$ can be expressed in terms of the GT strength function $S_{G T}$ according to

$$
\frac{\ln 2}{T_{1 / 2}}=\frac{\left(g_{A} / g_{V}\right)_{\mathrm{eff}}^{2}}{D} \sum_{E_{e x}=0}^{Q_{\beta}} f_{0}\left(Z, A, Q_{\beta}-E_{e x}\right) S_{G T}\left(E_{e x}\right) .
$$

For the phase-space volume $f_{0}$ as well as the $D$ factor and the vector and axial vector coupling constants (including the quenching factor), we refer to the work of [11]. For the $Q_{\beta}$ mass differences, we consider experimental values, whenever available or the D1M predictions, otherwise. 


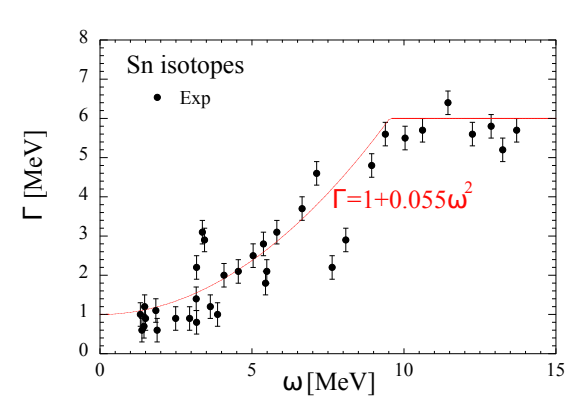

Figure 3. The experimental GT widths of Sn isotopes [8] and their correspondent parameterization.

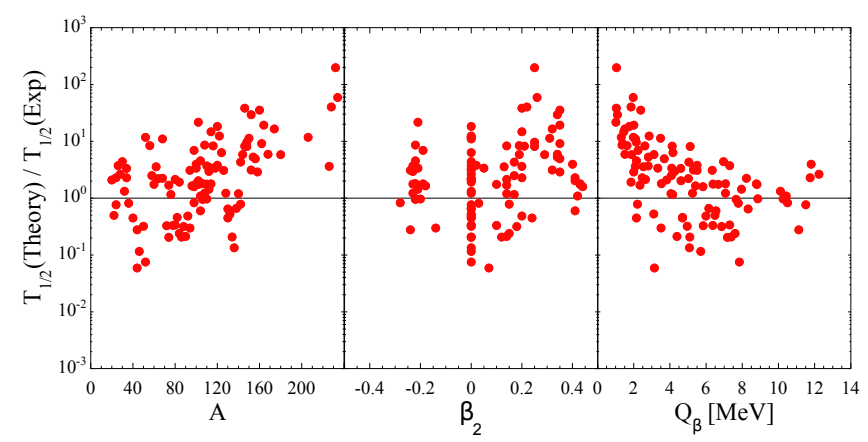

Figure 4. (color online) Ratio between the pnQRPA and experimental $\beta^{-}$-decay half-lives as a function of $A, \beta_{2}$ and $Q_{\beta}$.

The pnQRPA calculation provide, as shown in previous figures, a discrete strength distribution. In order to derive a smooth continuous strength function, the pnQRPA GT strength is folded with a Lorentz function, as classically done. We have choosen to parametrize the spreading width $\Gamma$ as shown in Fig. 3 in order to reproduce the experimental GT widths found experimentally in Sn isotopes [8].

To give an idea of the global predictions of our model, we compare in Fig.4 the pnQRPA $\beta^{-}$decay half-lives with experimental data. The results are plotted as a function of the mass number, the deformation parameter and the $Q_{\beta}$ value. They turn to be quite homogeneous with respect to $A$ and $\beta_{2}$. Larger deviations are found for nuclei close to the valley of $\beta$-stability (low $Q_{\beta}$ value), as found in most models. Globally, devation with respect to experimental data rarely exceeds one order of magnitude.

In conclusion, we presented here a fully consistent pnQRPA approach using a finite-range Gogny force. We applied our model to the analysis of charge-exchange modes paying a special attention to the GT resonances. The crucial role of deformation, automatically included in our approach, was analyzed. The agreement with experiment is satisfactory both for the strength distribution and the $\beta^{-}$-decay half-lives.

\section{References}

[1] S. Goriely, S. Hilaire, M. Girod and S. Péru, Phys. Rev. Lett. 102, 242501 (2009).

[2] J. Decharge and D. Gogny,Phys. Rev. C 21, 1568 (1980).

[3] S. Péru and H. Goutte, Phys. Rev. C 77, 044313 (2008).

[4] S. Péru, G. Gosselin, M. Martini, M. Dupuis, S. Hilaire and J. -C. Devaux, Phys. Rev. C 83, 014314 (2011).

[5] M. Martini, S. Péru and M. Dupuis, Phys. Rev. C 83, 034309 (2011).

[6] M. Arnould, S. Goriely, T. Takahashi, Phys. Rep. 450, 97 (2007).

[7] T. Wakasa et al., Phys. Rev. C 55, 2909 (1997).

[8] K. Pham et al., Phys. Rev. C 51, 526 (1995).

[9] H. Akimune et al., Phys. Rev. C 52, 604 (1995).

[10] J. H. Thies et al., Phys. Rev. C 86, 014304 (2012).

[11] I. N. Borzov and S. Goriely, Phys. Rev. C 62, 035501 (2000). 